\title{
Comparison of digital and traditional thoracic drainage systems for postoperative chest tube management after pulmonary resection: A prospective randomized trial
}

\author{
Kazuya Takamochi, MD, ${ }^{\mathrm{a}}$ Shuko Nojiri, PhD, ${ }^{\mathrm{b}}$ Shiaki Oh, MD, ${ }^{\mathrm{a}}$ Takeshi Matsunaga, MD, ${ }^{\mathrm{a}}$ \\ Kota Imashimizu, MD, ${ }^{a}$ Mariko Fukui, MD, ${ }^{a}$ and Kenji Suzuki, MD ${ }^{\mathrm{a}}$
}

\section{ABSTRACT}

Objective: The objective of this study was to evaluate whether a digital thoracic drainage system (group D) is clinically useful compared with a traditional thoracic drainage system (group T) in chest tube management following anatomic lung resection.

Methods: Patients scheduled to undergo segmentectomy or lobectomy were prospectively randomized before surgery to group $\mathrm{D}$ or $\mathrm{T}$. A stratification randomization was performed according to the following air leak risk factors: age, sex, smoking status, and presence of emphysema and/or chronic obstructive pulmonary disease. The primary end point was the duration of chest tube placement.

Results: No statistically significant differences were found between groups $\mathrm{D}$ $(\mathrm{n}=135)$ and $\mathrm{T}(\mathrm{n}=164)$ with regard to the duration of chest tube placement (median, 2.0 vs 3.0 days; $P=.149$ ), duration of hospitalization (median, 6.0 vs 7.0 days; $P=.548)$, or frequency of postoperative adverse events $(25.1 \%$ vs $20.7 \% ; P=.361)$. In subgroup analyses of the 64 patients with postoperative air leak (20 in group D and 44 in group T), the duration of chest tube placement (median, 4.5 vs 4.0 days; $P=.225$ ) and duration of postoperative air leak (median, 3.0 vs 3.0 days; $P=.226$ ) were not significantly different between subgroups.

Conclusions: The use of a digital thoracic drainage system did not shorten the duration of chest tube placement in comparison to a traditional thoracic drainage system after anatomic lung resection. (J Thorac Cardiovasc Surg 2018;155:1834-40)

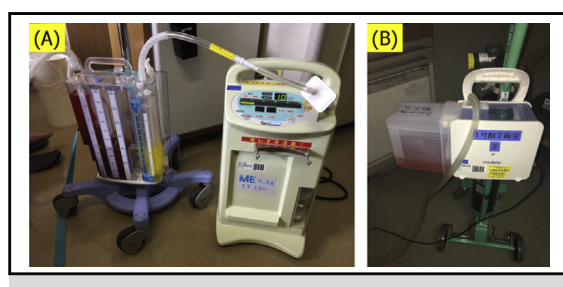

A, Traditional thoracic drainage system. B, Digital thoracic drainage system.

\section{Central Message}

The use of a digital thoracic drainage system did not shorten the duration of chest tube placement in comparison to a traditional thoracic drainage system after anatomic lung resection.

\section{Perspective}

We did not observe superiority of a digital thoracic drainage system for chest tube management after pulmonary resection compared with a traditional system. A well-planned, prospective randomized study limited to patients who develop a postoperative air leak is warranted to elucidate the effect of earlier air leak resolution using a digital monitoring system.

See Editorial Commentary page 1841.
From the a Department of General Thoracic Surgery, Juntendo University School of Medicine, Tokyo, Japan; and 'buntendo Clinical Research Support Center, Juntendo University School of Medicine, Tokyo, Japan.

Supported by Medela. The sponsor had no control over the interpretation, writing, or publication of this work. Support was also received in the form of a Grant-in-Aid for Cancer Research from the Ministry of Health, Labor, and Welfare of Japan and the Smoking Research Foundation.

Read at the 97th Annual Meeting of The American Association for Thoracic Surgery, Boston, Massachusetts, April 29-May 3, 2017.

Received for publication May 8, 2017; revisions received Aug 28, 2017; accepted for publication Sept 30, 2017; available ahead of print Dec 6, 2017.

Address for reprints: Kazuya Takamochi, MD, Department of General Thoracic Surgery, Juntendo University School of Medicine, 1-3, Hongo 3-chome, Bunkyo-ku, Tokyo 113-8431, Japan (E-mail: ktakamo@juntendo.ac.jp).

0022-5223/\$36.00

Copyright (c) 2017 by The American Association for Thoracic Surgery

https://doi.org/10.1016/j.jtcvs.2017.09.145
Chest tube management remains a critical aspect of the postoperative course for patients who undergo lung resection. The old dictum of no space, no problem is a guiding principle for chest drainage. ${ }^{1}$ Therefore, proper chest tube drainage of pleural effusion, bleeding, and air leakage is important to achieving adequate expansion of the remaining lung. However, solid evidence is lacking in all aspects of

Scanning this $\mathrm{QR}$ code will take you to a supplemental video. 


\section{Abbreviations and Acronyms \\ COPD $=$ chronic obstructive pulmonary disease \\ FEV1 = forced expiratory volume in 1 second \\ PAL = prolonged air leak}

chest tube management using a traditional thoracic drainage system, including whether external suction or water seal is effective to resolve postoperative air leaks. ${ }^{2}$ In clinical practice, decision making for chest tube management is conventionally performed based on the experiences of individual surgeons. The biggest weakness of traditional thoracic drainage systems is that they measure and grade air leaks in a subjective manner; therefore, interobserver disagreement on the presence of an air leak is frequent, ${ }^{3}$ even among experienced surgeons. Consequently, especially in patients with small air leaks, it is occasionally difficult to diagnose the presence or absence of air leak. In such cases, the chest tube must undergo a clamping trial before removal to rule out the existence of small occult air leaks.

In response to these concerns, the Thopaz (Medela Healthcare, Baar, Switzerland) pleural drainage system was developed, which incorporates digital sensors to accurately measure air flow (ie, amount of air leakage) and pleural pressure through the chest tube in $\mathrm{mL} / \mathrm{min}$ and $\mathrm{cm} \mathrm{H}_{2} \mathrm{O}$, respectively. ${ }^{4}$ The rate of air leakage can be observed on a display in real time. Digital surveillance for air leaks has been reported to reduce interobserver disagreement in decision making regarding the management of patients with chest tubes. ${ }^{3}$ Recently, we reported the clinical utility of objective chest tube management after pulmonary resection using a digital drainage system. ${ }^{5}$ Digital monitoring of peak air leakage and patterns of air leakage enable the prediction of prolonged air leak (PAL) after pulmonary resection. ${ }^{5}$ Information on the disappearance of air leaks could be derived from both the change in the rate of air leakage and the increase in fluctuation of pleural pressure. ${ }^{5}$

Several prospective, randomized studies have shown significantly earlier chest tube removal associated with the use of a digital thoracic drainage system compared with a traditional thoracic drainage system after lung resection. ${ }^{6-9}$ However, these studies employed different digital drainage devices, and their chest tube management protocols demonstrated considerable heterogeneity in terms of the use of suction, number of chest tubes, and assessment of chest tubes. Therefore, whether digital thoracic drainage can shorten the duration of air leakage or the duration of chest tube placement remains a matter of debate.

The objective of this study was to evaluate whether a digital thoracic drainage system (the Thopaz) is clinically useful compared with a traditional thoracic drainage system for chest tube management following anatomic lung resection in a prospective, randomized controlled trial.

\section{MATERIALS AND METHODS \\ Study Design}

This open-label, single-center, prospective, randomized, stratified, controlled trial was conducted to evaluate the superiority of treatment using a digital thoracic drainage system (group D) in comparison to a traditional thoracic drainage system (group T) in chest tube management after lung resection (Figure 1). This study was performed at Juntendo University Hospital between February 2015 and January 2016 after obtaining approval from the institutional review board of Juntendo University School of Medicine. Clinical trial registry No. UMIN: 000016715.

The primary end point was the duration of drain placement. The secondary end points were duration of postoperative air leak, frequency of PAL ( $\geq 5$ days), frequency of postoperative pleurodesis, days of hospitalization, and occurrence of postoperative adverse events.

\section{Patient Selection}

Patients who were scheduled to undergo anatomic lung resection (segmentectomy or lobectomy, excluding pneumonectomy) were eligible for inclusion in the study. Other eligibility criteria were $>800 \mathrm{~mL}$ predicted postoperative forced expiratory volume in 1 second (FEV1), where predicted postoperative FEV1 = preoperative FEV1 $\times$ (number of segments number of segments to be resected)/number of segments), performance status 0 or 1 (Eastern Cooperative Oncology Group classification), and adequate organ function. Written informed consent was obtained from all patients before surgery. The exclusion criteria were history of thoracotomy, active bacterial or fungal infection, ongoing systemic steroid administration (intravenous or oral), presence of uncontrolled diabetes mellitus, need for perioperative continuous intravenous heparin infusion, presence of a psychiatric disorder or psychological symptoms, and judged unsuitable for study participation by the investigator for any other reason.

\section{Randomization}

Eligible patients were randomized before surgery to receive chest tube management with a digital thoracic drainage system (group D) or a traditional thoracic drainage system (group T). The stratification randomization was performed using the stratification schedule created by the SAS statistical software package (version 9.4, SAS Institute Inc, Cary, NC) according to the following air leak risk factors: age ( $<70$ years vs $\geq 70$ years), sex (male vs female), smoking status (smoking $<5$ pack-years vs $\geq 5$ packyears), and emphysema and/or chronic obstructive pulmonary disease (COPD) (presence vs absence). Emphysema was defined as $>25 \%$ emphysematous change in the entire lung field on computed tomography

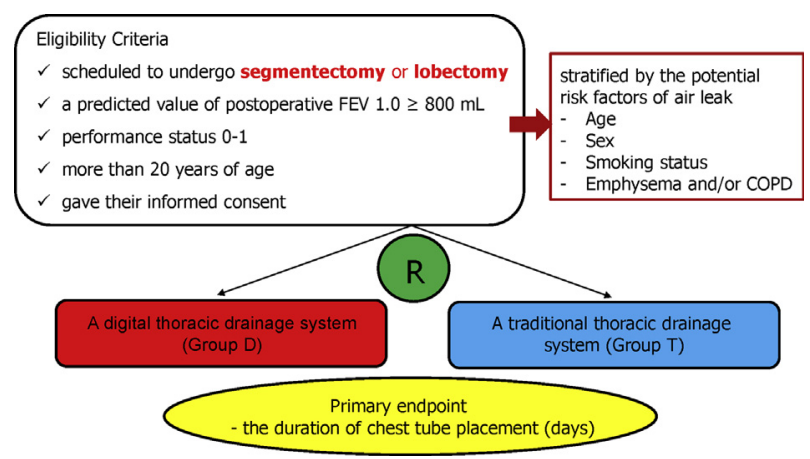

FIGURE 1. Protocol schema. FEV, Forced expiratory volume; COPD, chronic obstructive pulmonary disease. 
scanning. COPD was defined as FEV1\% (FEV1/forced vital capacity $\times 100)<70 \%$ on a pulmonary function test.

\section{Treatment}

Each surgical procedure was performed by attending thoracic surgeons or resident physicians under the supervision of an attending thoracic surgeon. The operative technique was standardized for all surgeons.

Segmentectomy or lobectomy was performed via posterolateral, anterolateral, or axillary open thoracotomy in all patients. We typically performed lobectomy and systematic mediastinal lymph node dissection for patients with radiologically invasive (ie, solid dominant) lung cancers. Mediastinal lymph node dissection was omitted for patients with ground-glass opacity-dominant nodules on thin-slice computed tomography scan that were highly suspected to be less invasive lung adenocarcinoma without lymph node involvement ${ }^{10,11}$ and for high-risk patients with any severe comorbidities. In those patients, segmentectomy was commonly performed to preserve pulmonary function when a sufficient surgical margin was obtained.

We used conventional automatic surgical staplers for developing the intersegmental plane at segmentectomy, dividing incomplete fissures at lobectomy, and closing the bronchus. The size of cartridges was selected according to the thickness and firmness of the lung parenchyma. A buttressed stapler (Endo GIA Tri-Staple Reinforce, Covidien Inc, Tokyo, Japan) was used to divide severely emphysematous lung parenchyma.

After the complete expansion of the remaining lung by an anesthesiologist, an intraoperative air leak test $\left(20 \mathrm{~cm} \mathrm{H}_{2} \mathrm{O}\right.$ applied pressure) was performed using a sterile saline solution. The amount of intraoperative air leakage was evaluated according to the following scoring system: grade $0=$ no detectable air bubbles, $1=$ countable air bubbles, $2=$ stream of bubbles, and 3 = coalesced bubbles (ie, air bubbles joining to form a continuous stream of air). ${ }^{12}$ Additional suture repair was applied when an air leak was detected. Sealant materials such as fibrin glues, synthetic sealants, and collagen patches coated with fibrinogen and thrombin were used if an air leak persisted (Video 1). Further air leak testing was not performed after sealant materials were used.

The patients in both groups were checked for the presence of an air leak twice daily by residents and attending thoracic surgeons, during the morning and evening rounds. In group $\mathrm{D}$, the digital thoracic drainage system was set at a regulated pressure level of $-10 \mathrm{~cm} \mathrm{H}_{2} \mathrm{O}$ from immediately after the operation until chest tube removal. The chest tubes were removed when the air leakage rate was $<20 \mathrm{~mL} /$ minute for $>12$ hours and the pleural effusion drainage volume was $\leq 300 \mathrm{~mL} /$ day. In group T, the chest drain was attached to a Mera Sucuum MS-008EX chest drainage system (Senko Medical, Tokyo, Japan) immediately after surgery. The chest tube was suctioned $\left(-10 \mathrm{~cm} \mathrm{H}_{2} \mathrm{O}\right.$ ) continuously until the morning of the first postoperative day and thereafter managed on a water seal. The chest tube was resuctioned at a level of $-10 \mathrm{~cm} \mathrm{H}_{2} \mathrm{O}$ only when checking for the presence of an air leak. The chest tubes were removed when no air leak was evident (ie, no bubbling observed in the water seal chamber at $-10 \mathrm{~cm} \mathrm{H}_{2} \mathrm{O}$ external suction), and when the pleural effusion drainage volume was $<300 \mathrm{~mL} /$ day. If an air leak was suspected, the chest tube was removed after a 24-hour chest tube clamping trial.

Among patients with air leaks continuing for $\geq 5$ days, pleurodesis via intrathoracic administration of the sclerosing agent OK432 with or without $50 \mathrm{~mL}$ autologous blood through the chest tube was performed according to the clinical judgment of the attending physician for each patient. ${ }^{1.3}$

\section{Sample Size Calculation}

The duration of chest tube placement in group $\mathrm{T}$ was defined as $4.5 \pm 2.52$ days. $^{12}$ A reduction of the duration of chest tube placement by 1 day was considered to be clinically relevant. Using a statistical software program, a power-based analysis was conducted at a 2 -sided significance level $(\alpha)$ of 0.05 and a power $(1-\beta)$ of 0.9 , which resulted in a value of

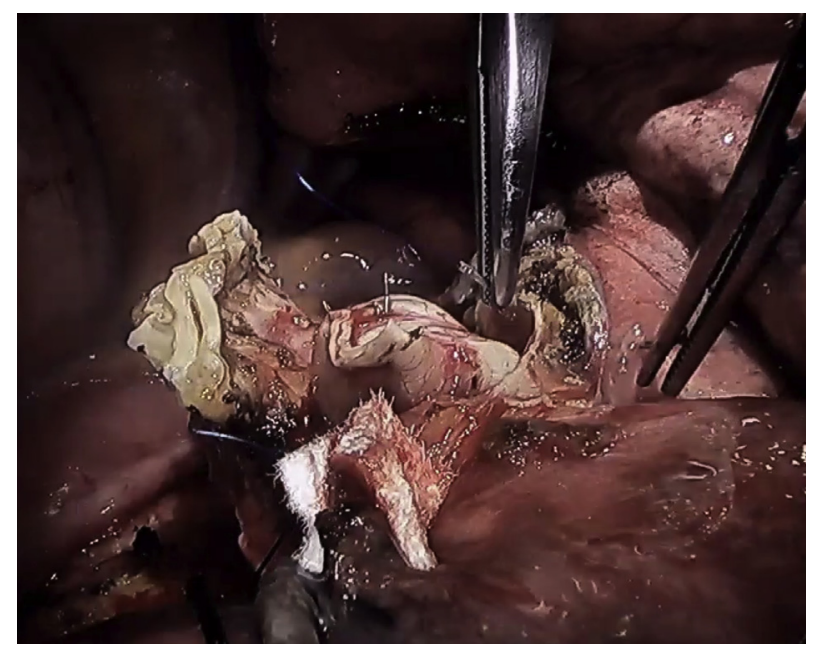

VIDEO 1. Intraoperative surgical procedures for air leak. Video available at: http://www.jtcvsonline.org/article/S0022-5223(17)32487-X/fulltext.

133. Therefore, the adequate number of subjects to be enrolled in the study was determined to be 150 per group, based on the assumption that the rate of withdrawal from the study would be approximately $10 \%$ of the enrolled subjects.

\section{Data Management and Statistical Analysis}

All data from enrolled patients were registered to the Research Electronic Data Capture system (https://projectredcap.org/software/), which is a secure Web application for building and managing online surveys and databases. Patient characteristics, intraoperative findings, and surgical outcomes including the primary and secondary endpoints were compared between group $\mathrm{D}$ and $\mathrm{T}$ using the $\chi^{2}$ test, Fisher exact test, Wilcoxon test, or $t$ test for statistical analysis. A multivariate analysis was performed using the Cox proportional hazards model to identify risk factors for the prolongation of chest tube placement. All statistical analyses were performed using the SAS statistical software package.

\section{RESULTS}

Of 320 eligible patients, 20 (17 undergoing wedge resection and 3 undergoing exploratory thoracotomy) were excluded due to protocol deviation after allocation (11 in group $\mathrm{D}$ and 9 in group $\mathrm{T}$ ), and 1 patient in group $\mathrm{T}$ was excluded from statistical analyses because reoperation

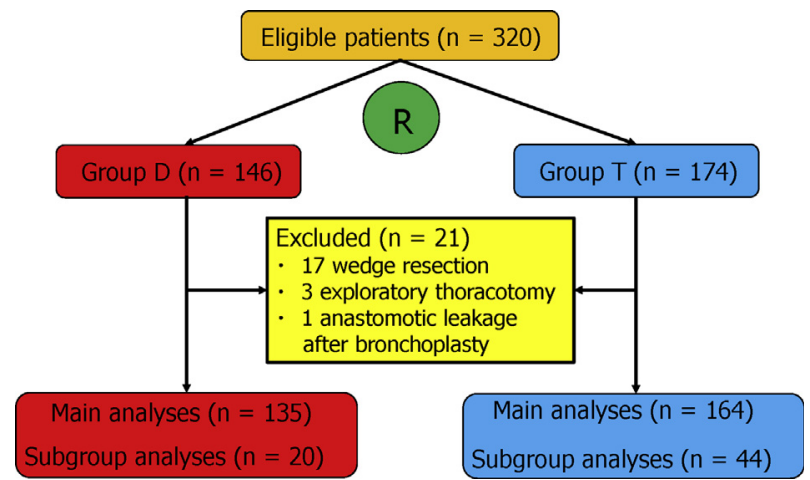

FIGURE 2. Flowchart of patient enrollment in the study. 
TABLE 1. Patient characteristics

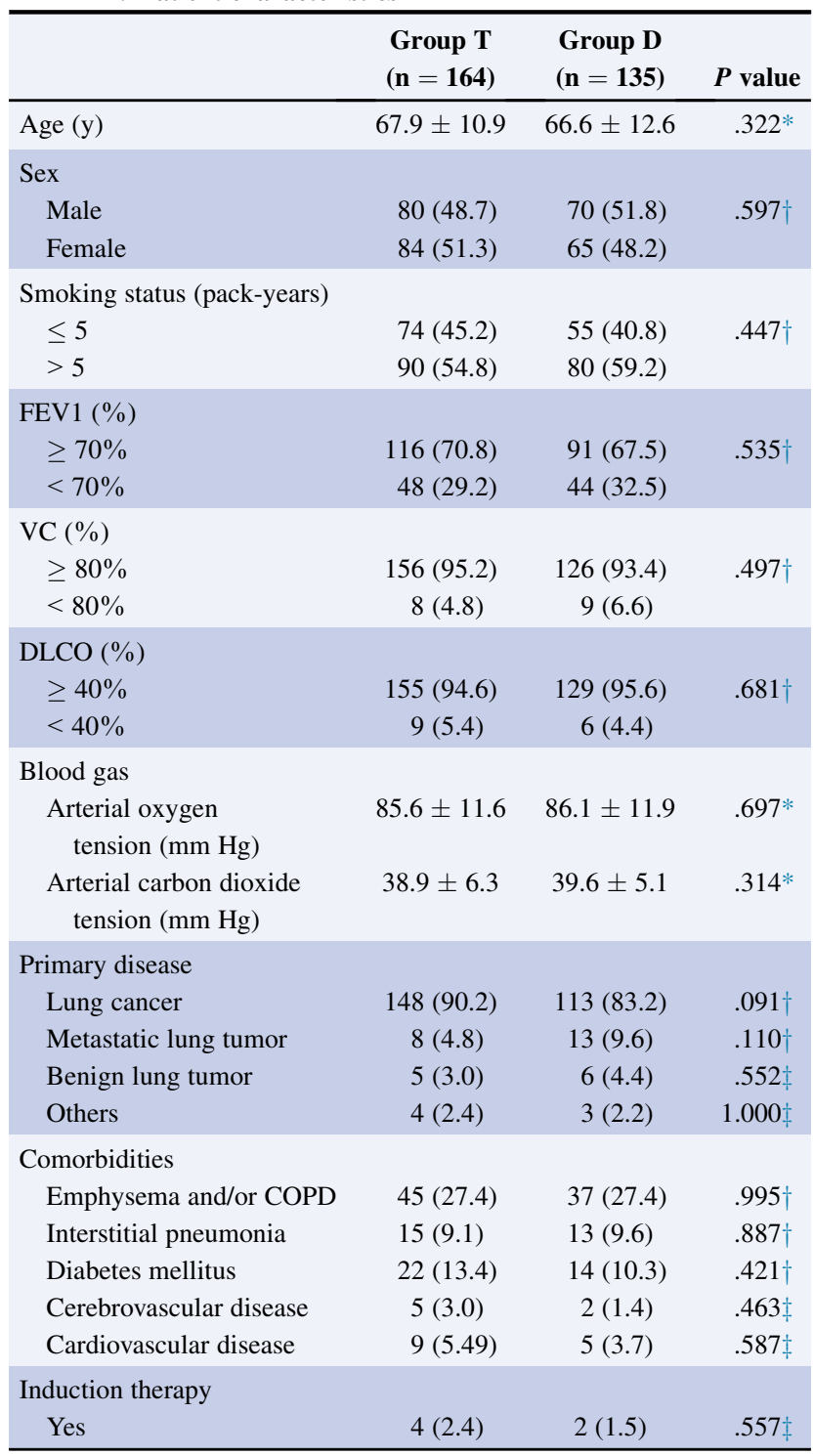

Values are presented as mean \pm standard deviation or $\mathrm{n}(\%) . F E V I$, Forced expiratory volume in 1 second; $V C$, vital capacity; $D L C O$, diffusing capacity of the lung for carbon monoxide; $C O P D$, chronic obstructive pulmonary disease. ${ }^{*} t$ test. $\dagger \chi^{2}$ test. $\ddagger$ Fisher exact test.

was performed due to massive anastomotic air leakage after bronchoplasty on postoperative day 1 . Therefore, the final patient numbers for analyses were 135 in group D and 164 in group T (Figure 2). There was no crossover between the groups due to patient request or problems with either of the systems.

Patient characteristics are shown in Table 1. The groups did not significantly differ in terms of age, sex, smoking status, FEV1, vital capacity, diffusing capacity of the lung for carbon monoxide, arterial oxygen tension, arterial carbon dioxide tension, primary disease, or comorbidities. Induction therapy was performed for a total of 6 patients (1 patient undergoing chemotherapy and 1 patient
TABLE 2. Comparisons of intraoperative findings between groups

\begin{tabular}{|c|c|c|c|}
\hline & $\begin{array}{l}\text { Group T } \\
(n=164)\end{array}$ & $\begin{array}{l}\text { Group D } \\
(\mathbf{n}=135)\end{array}$ & $P$ value \\
\hline \multicolumn{4}{|l|}{ Grade of adhesions } \\
\hline None & $113(68.9)$ & $99(73.3)$ & $.580^{*}$ \\
\hline Mild & $36(21.9)$ & $25(18.5)$ & \\
\hline Moderate & $11(6.7)$ & $10(7.4)$ & \\
\hline Severe & $4(2.4)$ & $1(0.7)$ & \\
\hline Length of skin incision $(\mathrm{cm})$ & $14.2 \pm 0.3$ & $13.8 \pm 0.4$ & $.431 \dagger$ \\
\hline \multicolumn{4}{|l|}{ Extent of pulmonary resection } \\
\hline Segmentectomy & $31(18.9)$ & $31(22.9)$ & $.389^{*}$ \\
\hline Lobectomy & $133(81.1)$ & $104(77.0)$ & \\
\hline \multicolumn{4}{|l|}{$\begin{array}{l}\text { Mediastinal lymph node } \\
\text { dissection }\end{array}$} \\
\hline Performed & $99(61.3)$ & $70(51.8)$ & $.139 *$ \\
\hline \multicolumn{4}{|l|}{ Number of chest tubes } \\
\hline 1 & $163(99.4)$ & $135(100)$ & $.364^{*}$ \\
\hline 2 & $1(0.6)$ & 0 & \\
\hline \multicolumn{4}{|l|}{ Grade of air leak } \\
\hline 0 & $82(50)$ & $74(54.8)$ & $.514^{*}$ \\
\hline 1 & $50(30.4)$ & $33(24.4)$ & \\
\hline 2 & $29(17.6)$ & $23(17.0)$ & \\
\hline 3 & $3(1.8)$ & $5(3.7)$ & \\
\hline \multicolumn{4}{|l|}{ Sealant use } \\
\hline Yes & $137(83.5)$ & $104(77.0)$ & $.157^{*}$ \\
\hline Fibrin glue & $20(12.2)$ & $22(16.3)$ & $.310^{*}$ \\
\hline $\begin{array}{l}\text { Fibrinogen- and thrombin- } \\
\text { coated collagen patches }\end{array}$ & $11(6.7)$ & $3(2.2)$ & $.097 \ddagger$ \\
\hline $\begin{array}{l}\text { Combined use of fibrin glues } \\
\text { and synthetic sealant }\end{array}$ & $106(64.6)$ & $78(57.8)$ & $.225^{*}$ \\
\hline
\end{tabular}

undergoing chemoradiation in group $\mathrm{D}$ and 1 patient undergoing chemotherapy and 3 patients undergoing chemoradiation in group $\mathrm{T}$ ). The proportion of patients who underwent induction therapy was not significantly different between the groups $(1.5 \%$ in group $\mathrm{D}$ and $2.4 \%$ in group $\mathrm{T}$; $P=.557)$.

Intraoperative findings, including grade of adhesion, surgical procedures (eg, length of skin incision, extent of pulmonary resection, and mediastinal lymph node dissection), number of chest tubes, grade of air leak, and sealant use also did not significantly differ between the groups (Table 2).

Surgical outcomes of all patients are shown in Table 3. No statistically significant differences were found between groups $\mathrm{D}$ and $\mathrm{T}$ in terms of the duration of chest tube placement (median, 2.0 days vs 3.0 days; $P=.149$ ), incidence of PAL $\geq 5$ days ( $7.4 \%$ vs $7.9 \% ; P=.867)$, duration of postoperative hospital stay (median, 6.0 days vs 7.0 days; $P=.548)$, and frequency of postoperative adverse events ( $25.1 \%$ vs $20.7 \% ; P=.361)$. The frequency of performing a chest tube clamping trial before its removal was significantly lower in group D than in group $\mathrm{T}(0.7 \%$ vs $35.3 \%$; 
TABLE 3. Comparisons of surgical outcomes between groups

\begin{tabular}{|c|c|c|c|}
\hline & $\begin{array}{l}\text { Group T } \\
(n=164)\end{array}$ & $\begin{array}{l}\text { Group D } \\
(\mathbf{n}=135)\end{array}$ & $\begin{array}{c}P \\
\text { value } \\
\end{array}$ \\
\hline Postoperative air leak & $44(26.8)$ & $20(14.8)$ & $.012 *$ \\
\hline $\begin{array}{l}\text { Duration of chest tube } \\
\text { placement (d) }\end{array}$ & $3.0(2.0-4.0)$ & $2.0(2.0-3.0)$ & $.149 \dagger$ \\
\hline Prolonged air leak $(\geq 5 \mathrm{~d})$ & $13(7.9)$ & $11(8.1)$ & $.944 *$ \\
\hline Clamping test of a chest tube & $58(35.3)$ & $1(0.7)$ & $<.001 \ddagger$ \\
\hline \multicolumn{4}{|l|}{$\begin{array}{l}\text { Determining factor for } \\
\text { maintaining chest tube } \\
\text { placement }\end{array}$} \\
\hline Presence of an air leak & $31(18.9)$ & $15(11.1)$ & $.063 \dagger$ \\
\hline Amount of pleural effusion & $133(81.1)$ & $120(88.9)$ & \\
\hline $\begin{array}{l}\text { Duration of postoperative } \\
\text { hospital stay (d) }\end{array}$ & $7.0(6.0-8.0)$ & $6.0(6.0-8.0)$ & $.548 \dagger$ \\
\hline \multicolumn{4}{|l|}{ Postoperative adverse events } \\
\hline Total & $34(20.7)$ & $34(25.1)$ & $.361 *$ \\
\hline Pneumonia & $1(0.6)$ & $1(0.7)$ & \\
\hline Atelectasis & $1(0.6)$ & $5(14.7)$ & \\
\hline Bleeding & $1(0.6)$ & $1(0.7)$ & \\
\hline Arrhythmia & $12(7.3)$ & $10(7.4)$ & \\
\hline Chylothorax & $2(1.2)$ & $1(0.7)$ & \\
\hline Others & $17(10.3)$ & $135(11.8)$ & \\
\hline
\end{tabular}

Values are presented as $\mathrm{n}(\%)$ or median (interquartile range). ${ }^{*} \chi^{2}$ test. $\dagger$ Wilcoxon test. ‡๋Fisher exact test.

$P<.001)$. The incidence of postoperative air leak immediately after surgery was significantly higher in group $\mathrm{T}$ than in group $\mathrm{D}(26.8 \%$ vs $14.8 \% ; P=.012)$. Although the presence of an air leak was a more frequent determining factor for the decision to maintain chest tube placement than the amount of pleural effusion in group $\mathrm{T}$ than in group $\mathrm{D}$, the difference was not statistically significant $(18.9 \%$ vs $11.1 \% ; P=.063)$. No patients required chest tube reinsertion due to recurrent pneumothorax in either group.

Subgroup analyses of the 64 patients who had a postoperative air leak (20 in group D and 44 in group T, defined as subgroups $\mathrm{D}$ and $\mathrm{T}$, respectively) were then performed (Table 4). The duration of chest tube placement (median, 4.5 days vs 4.0 days; $P=.225$ ), duration of postoperative air leak (median, 3.0 days vs 3.0 days; $P=.226$ ), duration of postoperative hospital stay (median, 9.5 days vs 8.0 days; $P=.208$ ), and frequency of postoperative adverse events $(20.0 \%$ vs $34.1 \% ; P=.403)$ were not significantly different between subgroups $\mathrm{D}$ and $\mathrm{T}$. The frequency of pleurodesis was significantly higher in subgroup $\mathrm{D}$ than in subgroup T $(35.0 \%$ vs $6.8 \% ; P=.008)$. The frequency of performing a chest tube clamping trial was significantly lower in subgroup D than in subgroup T $(0 \%$ vs $52.2 \%$; $P<.001)$.

A multivariate analysis was performed to identify risk factors for the prolongation of chest tube placement using a Cox proportional hazards model that included age, sex, smoking status, emphysema and/or COPD (presence vs
TABLE 4. Comparisons of surgical outcomes between the subgroups of patients with postoperative air leak

\begin{tabular}{lccr}
\hline & $\begin{array}{c}\text { Subgroup T } \\
(\mathbf{n}=\mathbf{4 4})\end{array}$ & $\begin{array}{c}\text { Subgroup D } \\
(\mathbf{n}=\mathbf{2 0})\end{array}$ & $\boldsymbol{P}$ value \\
\hline Duration of air leak (d) & $3.0(1.5-4.0)$ & $3.0(2.0-6.5)$ & $.226^{*}$ \\
$\begin{array}{l}\text { Duration of chest tube } \\
\text { placement (d) }\end{array}$ & $4.0(3.0-5.0)$ & $4.5(3.0-8.0)$ & $.225^{*}$ \\
\hline Prolonged air leak ( $\geq 5 \mathrm{~d})$ & $7(15.9)$ & $6(30.0)$ & $.194 \dagger$ \\
Clamping test of a chest tube & $23(52.2)$ & $0(0)$ & $<.001 \dagger$ \\
\hline Pleurodesis & $3(6.8)$ & $7(35.0)$ & $.008 \ddagger$ \\
$\begin{array}{l}\text { Duration of postoperative } \\
\quad \text { hospital stay (d) }\end{array}$ & $8.0(6.0-10.0)$ & $9.5(6.0-13.0)$ & $.208^{*}$ \\
\hline Postoperative adverse events & $15(34.1)$ & $9(20.0)$ & $.403 \dagger$ \\
\hline
\end{tabular}

Values are presented as $\mathrm{n}(\%)$ or median (interquartile range). $*$ Wilcoxon test. $\dagger \chi^{2}$ test. †ेFisher exact test.

absence), type of primary disease (lung cancer vs other), mediastinal lymph node dissection (performed vs not performed), extent of pulmonary resection (lobectomy vs segmentectomy), sealant use (yes vs no), determining factor for maintaining chest tube placement (presence of an air leak vs amount of pleural effusion), and type of thoracic drainage system (traditional vs digital). Only the determining factor for maintaining chest tube placement was significantly associated with duration of chest tube placement. In comparison to the amount of pleural effusion, the hazard ratio for the presence of an air leak was 1.89 for the prolongation of chest tube placement $(P<.001)$ (Table 5).

\section{DISCUSSION}

It has been hypothesized that the duration of chest tube placement may be shortened with the use of a digital thoracic drainage system via the following mechanisms. First, the duration of air leak may be shortened by stable pleural pressure control with a digital thoracic drainage system. ${ }^{5}$ Second, the objective evaluation of air leak resolution by a digital thoracic drainage system may reduce the likelihood of interobserver disagreement and obviates the empirical need for clamping trial of a chest tube before removal. ${ }^{3}$ As a result, earlier judgment for the timing of chest tube removal may be possible. However, the present study did not demonstrate that the use of a digital thoracic drainage system shortened the duration of chest tube placement in comparison to a traditional thoracic drainage system.

There are several possible explanations for the negative results of the present study. First, the number of patients who developed postoperative air leak was too small to evaluate the positive effect of air leak resolution by a digital thoracic drainage system. Furthermore, there was a statistically significant bias in the number of patients with postoperative air leak (20 in group D and 44 in group T; $P=.012$ ). Therefore, the results may be substantially skewed because the presence or absence of a postoperative air leak is considered to have a significant influence on the duration of chest 
TABLE 5. Multivariate analysis of risk factors for the prolongation of chest tube placement

\begin{tabular}{|c|c|c|c|}
\hline & $\begin{array}{l}\text { Hazard } \\
\text { ratio }\end{array}$ & $\begin{array}{l}\text { 95\% Confidence } \\
\text { interval }\end{array}$ & $P$ value* \\
\hline Age $\geq 70(y)($ ref, $<70$ y) & 1.14 & $0.90-1.44$ & .294 \\
\hline Male sex (ref, female) & 0.85 & $0.65-1.13$ & .269 \\
\hline Smoking status $\geq 5$ pack-years (ref, $<5$ pack-years) & 1.04 & $0.76-1.41$ & .822 \\
\hline Presence of emphysema/chronic obstructive pulmonary disease, presence (ref, absence) & 0.91 & $0.68-1.22$ & .517 \\
\hline Lung cancer (ref, other disease) & 0.83 & $0.58-1.21$ & .334 \\
\hline Presence of mediastinal lymph node dissection (ref, absence) & 1.02 & $0.77-1.34$ & .913 \\
\hline Extent of pulmonary resection is lobectomy (ref, segmentectomy) & 0.79 & $0.58-1.09$ & .146 \\
\hline Sealant use (ref, no sealant use) & 0.90 & $0.69-1.22$ & .495 \\
\hline $\begin{array}{l}\text { Determining factor for maintaining chest tube placement was presence of an air leak (ref, amount of } \\
\text { pleural effusion) }\end{array}$ & 1.89 & $1.36-2.62$ & $<.001$ \\
\hline Digital thoracic drainage system (ref, traditional system) & 1.03 & $0.82-1.30$ & .774 \\
\hline
\end{tabular}

ref, Reference category (hazard ratio, 1). *Cox proportional hazards model.

tube placement. Second, clinical judgment for chest tube removal was performed once per day at morning rounds, because chest tubes were removed in the daytime. Objective real-time data for air leakage cannot be assessed using a traditional thoracic drainage system. For the above reasons, any differences in hours of duration between the groups, if present, could not be evaluated, which may result in the underestimation of the effectiveness of a digital thoracic drainage system. Third, the criteria for chest tube removal used in this study were based not only on the absence of air leakage but also on the amount of pleural effusion drainage ( $\leq 300 \mathrm{~mL} /$ day). Because the number of patients who developed a postoperative air leak was small, the judgment of chest tube removal was based on the amount of pleural effusion drainage for a substantial proportion of patients. However, in the present study, the presence of an air leak was significantly associated with the prolongation of chest tube placement in comparison to the amount of pleural effusion (hazard ratio, 1.89; $P<.001$ ) on multivariate analysis. Recently, the total amount of pleural effusion was reported not to significantly differ between traditional and digital drainage system groups $(\mathrm{n}=50$ and $\mathrm{n}=53$, respectively) following oncologic pulmonary resection in a prospective randomized trial. ${ }^{14}$ These findings support our finding that the amount of pleural effusion is not critical to evaluating the duration of chest tube placement. Finally, although stratification randomization was used to avoid imbalance between the treatment groups in prognostic variables, there was a relatively small imbalance in the total number of patients in each group. However, we believe that the effect of this imbalance on the variance of effect measures is not critical. ${ }^{15}$

In a multicenter prospective randomized trial comparing digital monitoring using the Thopaz system to a traditional thoracic drainage system in 381 patients who underwent segmentectomy or lobectomy, Pompili and colleagues ${ }^{9}$ reported that patients managed with the Thopaz system experienced a shorter duration of chest tube placement and shorter hospital stay compared with those managed with traditional devices. By contrast, Gilbert and colleagues ${ }^{16}$ reported no statistically significant differences in the duration of chest tube drainage or hospital stay in both a group of 87 patients without air leak and a group of 85 patients with air leak in their single-center prospective randomized trial. Lijkendijk and colleagues ${ }^{17}$ also reported that the Thopaz system did not significantly reduce the duration of chest tube drainage or hospitalization compared with traditional water seal drainage. The outcomes of the present study are consistent with those of the latter 2 randomized studies. Therefore, the superiority of a digital thoracic drainage system in terms of the shorter duration of chest tube drainage and hospital stay remains unclear. A large-scale randomized study limited to patients who develop a postoperative air leak is warranted to elucidate the effect of earlier air leak resolution by a digital monitoring system compared with a traditional thoracic drainage system in this population.

The cost difference between the Thopaz system and the traditional thoracic drainage system was not assessed in the present clinical trial because a preplanned cost analysis was not performed. Cost-efficiency is an important requirement for an ideal chest drainage system. Therefore, a well-planned cost analysis considering many factors, such as the cost of disposable devices, cost of treatment for postoperative complications, cost of hospitalization, and the health care system of the country, is necessary to provide clear evidence related to cost differences in future studies.

\section{CONCLUSIONS}

The use of a digital thoracic drainage system did not shorten the duration of chest tube placement in comparison to a traditional thoracic drainage system. However, traditional thoracic drainage systems are likely to be replaced 
by digital thoracic drainage in the near future due to various clinical advantages. ${ }^{9,18}$ Further prospective studies are needed to establish reliable chest tube management protocols using a digital thoracic drainage system.

\section{Conflict of Interest Statement}

This work was supported by Medela. Authors have nothing to disclose with regard to commercial support.

\section{References}

1. Haithcock BE, Feins RH. Complications of pulmonary resections. In: Shields TW, LoCicero J, Reed CE, Feins RH, eds. General thoracic surgery. 7th ed. Philadelphia: Lippincott Williams \& Wilkins; 2009:551-9.

2. Qiu T, Shen Y, Wang MZ, Wang YP, Wang D, Wang ZZ, et al. External suction versus water seal after selective pulmonary resection for lung neoplasm: a systematic review. PLoS One. 2013;8:e68087.

3. McGuire AL, Petrcich W, Maziak DE, Shamji FM, Sundaresan SR, Seely AJ, et al. Digital versus analogue pleural drainage phase 1: prospective evaluation of interobserver reliability in the assessment of pulmonary air leaks. Interact Cardiovasc Thorac Surg. 2015;21:403-7.

4. Brunelli A, Salati M, Pompili C, Refai M, Sabbatini A. Regulated tailored suction vs regulated seal: a prospective randomized trial on air leak duration. Eur J Cardiothorac Surg. 2013;43:899-904.

5. Takamochi K, Imashimizu K, Fukui M, Maeyashiki T, Suzuki M, Ueda T, et al. Utility of objective chest tube management after pulmonary resection using a digital drainage system. Ann Thorac Surg. 2017;104:275-83.

6. Brunelli A, Salati M, Refai M, Di Nunzio L, Xiume F, Sabbatini A. Evaluation of a new chest tube removal protocol using digital air leak monitoring after lobectomy: a prospective randomised trial. Eur J Cardiothorac Surg. 2010;37:56-60.

7. Cerfolio RJ, Bryant AS. The benefits of continuous and digital air leak assessment after elective pulmonary resection: a prospective study. Ann Thorac Surg. 2008;86:396-401.

8. Mier JM, Fibla JJ, Molins L. The benefits of digital thoracic drainage system for outpatients undergoing pulmonary resection surgery. Rev Port Pneumol. 2011; 17:225-7.
9. Pompili C, Detterbeck F, Papagiannopoulos K, Sihoe A, Vachlas K, Maxfield MW, et al. Multicenter international randomized comparison of objective and subjective outcomes between electronic and traditional chest drainage systems. Ann Thorac Surg. 2014;98:490-6; discussion 6-7.

10. Kodama K, Higashiyama M, Yokouchi H, Takami K, Kuriyama K, Mano M, et al. Prognostic value of ground-glass opacity found in small lung adenocarcinoma on high-resolution CT scanning. Lung Cancer. 2001;33:17-25.

11. Suzuki K, Kusumoto M, Watanabe S, Tsuchiya R, Asamura H. Radiologic classification of small adenocarcinoma of the lung: radiologic-pathologic correlation and its prognostic impact. Ann Thorac Surg. 2006;81:413-9.

12. Takamochi K, Oh S, Miyasaka Y, Matsunaga T, Kitamura Y, Fukui M, et al. Prospective randomized trial comparing buttressed versus nonbuttressed stapling in patients undergoing pulmonary lobectomy. Thorac Cardiovasc Surg. 2014;62: 696-704.

13. Yokomise H, Satoh K, Ohno N, Tamura K. Autoblood plus OK432 pleurodesis with open drainage for persistent air leak after lobectomy. Ann Thorac Surg. 1998;65:563-5.

14. De Waele M, Agzarian J, Hanna WC, Schieman C, Finley CJ, Macri J, et al. Does the usage of digital chest drainage systems reduce pleural inflammation and volume of pleural effusion following oncologic pulmonary resection? A prospective randomized trial. J Thorac Dis. 2017;9:1598-606.

15. Lachin JM. Statistical properties of randomization in clinical trials. Control Clin Trials. 1988;9:289-311.

16. Gilbert S, McGuire AL, Maghera S, Sundaresan SR, Seely AJ, Maziak DE, et al. Randomized trial of digital versus analog pleural drainage in patients with or without a pulmonary air leak after lung resection. J Thorac Cardiovasc Surg. 2015;150:1243-9.

17. Lijkendijk M, Licht PB, Neckelmann K. Electronic versus traditional chest tube drainage following lobectomy: a randomized trial. Eur J Cardiothorac Surg. 2015;48:893-8; discussion 8 .

18. Rathinam S, Bradley A, Cantlin T, Rajesh PB. Thopaz portable suction systems in thoracic surgery: an end user assessment and feedback in a tertiary unit. J Cardiothorac Surg. 2011;6:59.

Key Words: digital thoracic drainage system, chest tube management, pulmonary resection 